\title{
"Un primer apunte sobre la Ley de cohesión y calidad del sistema nacional de salud"
}

\author{
Elsa Marina Álvarez González \\ Universidad de Málaga \\ Bicaria de Investigación
}

\begin{abstract}
SUMARIO: 1.- INTRODUCCIÓN. 2.- SISTEMA NACIONAL DE SALUD Y SISTEMA DE LA SEGURIDAD SOCIAL. 3.- PRESTACIONES DEL SISTEMA NACIONAL DE SALUD. 4.- LA COORDINACIÓN Y LA COOPERACIÓN EN EL SNS: EL CONSEJO INTERTERRITORIAL Y LA ALTA INSPECCIÓN. 5.- A MODO DE CONCLUSIÓN.
\end{abstract}

\section{INTRODUCCIÓN}

Hacer una valoración inmediata de la recién promulgada Ley 16/2003, de 28 de mayo, de Cohesión y Calidad del Sistema Nacional de Salud (en adelante, LCCSNS) puede resultar algo aventurado. Sin embargo, no podemos resistirnos a avanzar algunas reflexiones sobre la misma. No en vano, desde distintos sectores se venía reivindicado la necesidad apremiante de una Ley de este tipo para conseguir, una vez culminado el proceso de descentralización sanitaria -desde hace tan sólo dos años todas las Comunidades Autónomas (CCAA) tienen traspasadas las funciones y servicios para gestionar la asistencia sanitaria-, la definitiva implantación del modelo de sanidad pública que recogía la Ley General de Sanidad de 1986 (LGS).

La LGS proclamaba, aunque, a nuestro entender, de una forma un tanto desordenada, una concepción integral de la sanidad pública, que incluía dos grandes objetivos para la actividad administrativa en la sanidad: por un lado, la acción administrativa en materia de salud pública en general y, por otro, la acción prestacional o asistencial. A este último fin creó, por lo demás, el Sistema Nacional de Salud (SNS), entendido como el conjunto de los Servicios de Salud de la Administración General del Estado y de las Comunidades Autónomas, en el que se integrarían todas las estructuras y servicios públicos al servicio de la salud, así como las funciones y prestaciones que son responsabilidad de los poderes públicos, todo ello para garantizar el derecho a la protección de la salud reconocido en el art. 43.1 CE. 
Sobre esta base, la LGS preveía que una vez terminado el proceso de traspasos de la asistencia sanitaria a todas las CCAA, el Estado sería el encargado de desempeñar el papel de director y coordinador general del Sistema Nacional de Salud, a través de las competencias exclusivas que constitucionalmente tiene reservadas: sanidad exterior, bases y coordinación general de la sanidad, legislación sobre productos farmacéuticos y legislación básica de la Seguridad Social. A lo que debía añadirse el ejercicio de la Alta Inspección, no explícito en el texto constitucional, pero contemplado expresamente en todos los Estatutos de Autonomía y en la propia Ley General de Sanidad.

Ese proceso de descentralización sanitaria ha culminado hace bien poco, en concreto, a través de los últimos Reales Decretos de traspasos de los servicios, centros y funciones, es decir, los medios materiales y humanos existentes y necesarios para la gestión por las CCAA de la asistencia sanitaria, publicados los días 28 y 29 de diciembre de 2001, y la Ley 21/2001, de 27 de diciembre, en la que se establece el nuevo sistema de financiación autonómica. De esta manera, la gestión de la asistencia sanitaria por las CCAA, en principio asumida tan sólo por siete, las denominadas históricas, más Andalucía, Canarias, Comunidad Valenciana y Navarra, está ya en manos de todas ellas.

Desde ese momento, la realidad efectiva del sector sanitario es, pues, bien distinta de la que contempló la LGS de 1986 (para ésta la descentralización general aparecía en gran medida sólo como un horizonte de futuro), asumiendo consecuentemente la cooperación y la coordinación entre las distintas instancias públicas competentes y el Estado, como garante último de la cohesión en el conjunto del Sistema Nacional de Salud, un papel fundamental. Tales principios, junto a la calidad en la atención sanitaria -convertido en los últimos años en el motor de impulso y cambio del sistema-, aparecen como los principios fundamentales que inspiran la regulación de la LCCSNS.

Bajo estos principios, la Ley trata de reordenar el sistema sanitario readaptándolo a la nueva situación autonómica, es decir, de gestión por las CCAA de la asistencia sanitaria, contexto éste en el que cobran especial importancia una serie de medidas organizativas. Así el reconocimiento de mayor protagonismo a determinados órganos que si bien se crearon al amparo de la Ley General de Sanidad, sin embargo, no han tenido el desarrollo y funcionamiento previstos en aquel momento y necesitaban cobrar mayor fuerza y dinamismo, una redefinición de las competencias legales del Estado en el ámbito sanitario, y la creación de nuevos órganos, como el Observatorio del Sistema Nacional de Salud o la Agencia de Calidad Sanitaria, necesarios para alcanzar los ob- 
jetivos comunes de la Ley: la equidad, la calidad y la participación social en un sistema sanitario armónico y cohesionado.

En el presente trabajo nos limitamos a realizar un primer apunte sobre esta nueva regulación del sistema sanitario público, para valorar cómo será o podrá ser su aplicación y si los objetivos a que se refiere la exposición de motivos de la LCCSNS, con la regulación que posteriormente incorpora su texto normativo, son efectivamente alcanzables en la práctica o no. Hay que advertir en todo caso que esa regulación de la LCCSNS, aunque articula una reforma sanitaria, en ningún momento implica la sustitución de la Ley General de Sanidad, la cual sigue vigente salvo en los preceptos que expresamente aquélla deroga ${ }^{1}$. Algo natural, pues la LCCSNS no pretende tal sustitución, sino tan sólo regular los aspectos imprescindibles para configurar un Sistema Nacional de Salud cohesionado y de calidad, con todo lo que ello implica.

\section{SISTEMA NACIONAL DE SALUD Y SISTEMA DE LA SEGURI- DAD SOCIAL}

La nueva Ley de Cohesión y Calidad del Sistema Nacional Salud se ha promulgado al amparo del art. 149.1.16 $6^{\mathrm{a}}$ y $17^{\mathrm{a}} \mathrm{CE}$. De esta manera, la asistencia sanitaria sigue vinculada al Sistema de la Seguridad Social, cuestión no baladí, pues de la inclusión de la asistencia sanitaria en el Sistema Nacional de Salud o en la Seguridad Social se derivan consecuencias totalmente diferentes.

Situando el punto de partida en la CE, ésta recoge de forma separada las materias de Seguridad Social y de Sanidad: el artículo 41 se refiere a la existencia de un régimen público de Seguridad Social para todos los ciudadanos; y el artículo 43 reconoce el derecho a la salud y recoge la competencia de los poderes públicos para organizar y tutelar la salud pública a través de medidas preventivas y de las prestaciones y servicios necesarios. Por otra parte, dentro de la distribución competencial, el artículo 148.1.21 a reconoce la competencia de las CCAA en materia de sanidad e higiene; el artículo 149.1.16 $6^{\underline{a}}$ recoge la competencia exclusiva del Estado en materia de sanidad exterior, bases y coordinación general de la sanidad, y legislación sobre productos farmacéuticos; y el artículo 149.1.17 ${ }^{\mathrm{a}}$ se refiere a la legislación básica y régimen económico

\footnotetext{
${ }^{1}$ Según la Disposición Derogatoria Primera de la LCCSNS, los únicos preceptos de la LGS que quedan derogados son el artículo 43, que regula la Alta Inspección, y el artículo 47, que regula el Consejo Interterritorial del Sistema Nacional de Salud.
} 
de la Seguridad Social, sin perjuicio de la ejecución de sus servicios por las CCAA.

En consecuencia, a partir de la Constitución se produce una diferenciación entre la materia de salud pública, asumible por todas las CCAA, y la materia de asistencia sanitaria, que en la medida en que formara parte de la Seguridad Social -como era el caso en su momento- sólo podía ser asumida por las CCAA cuyos Estatutos de Autonomía hicieran referencia a la ejecución por aquéllas de los servicios propios de la Seguridad Social. Así, en efecto, reciben los traspasos de los servicios propios de la asistencia sanitaria inicialmente las llamadas CCAA “de vía rápida” (País Vasco, Cataluña, Galicia, Andalucía, Canarias, Comunidad Valenciana y Navarra), mientras que el resto de CCAA no podían recibir dichos traspasos al no haber asumido la competencia en materia de ejecución de los servicios de la Seguridad Social. Sólo mucho más tarde, cuando esta asunción se concreta con las reformas estatutarias que tienen lugar desde 1996 hasta 1999, estas últimas CCAA podrán prestar ya también la asistencia sanitaria, producidos los correspondientes traspasos de servicios.

La asistencia sanitaria se entendió, pues, vinculada conceptualmente a la materia Seguridad Social, por más que esa vinculación fuera más que discutible. Se ha señalado en este sentido ${ }^{2}$ que la CE no impone un modelo concreto sobre la prestación de los servicios sanitarios, y que no se puede deducir de la CE una obligada inclusión de la asistencia sanitaria dentro de la materia propia de la Seguridad Social. Más bien, esa conclusión derivaría de la consideración histórica en nuestro país de la prestación de la asistencia sanitaria como prestación propia de la Seguridad Social (el contenido que ésta deba tener no puede, por lo demás, deducirse del texto constitucional, tal y como ha subrayado la STC 206/1997, de 27 de noviembre).

No puede extrañar por ello mismo que, desde un criterio alejado de esa vinculación de la asistencia sanitaria y la Seguridad Social, la Ley General de Sanidad de 1986, al crear el Sistema Nacional de Salud como sistema único y a la vez descentralizado, basado en la universalidad y en la concepción integral de la sanidad, optara por unificar dentro del título competencial sanidad

\footnotetext{
${ }^{2}$ J. TORNOS MÁS, "Sistema de Seguridad Social versus Sistema Nacional de Salud”, Revista Derecho y Salud, Vol.10, Núm.1, Enero-Junio 2002; MUÑOZ MACHADO, Derecho público de las Comunidades Autónomas, Vol.I, Civitas, Madrid 1982; "La organización de los sistemas de salud", Primer Congreso de Derecho y Salud, Barcelona 1992; y La formación y crisis de los servicios sanitarios públicos, Alianza Ed. Madrid 1995, pág. 91 y ss.
} 
(artículo 149.1.16 $6^{\mathrm{a}} \mathrm{CE}$ ) toda la materia sanitaria, integrando así la asistencia sanitaria en el Sistema Nacional de Salud ${ }^{3}$. Esta línea se refuerza posteriormente, por lo demás, con la Ley 26/1990, de 20 de diciembre, de prestaciones no contributivas, que al disponer la refundición de las normas básicas de Seguridad Social, exceptuó de la refundición precisamente todo lo relativo a la asistencia sanitaria. Cumpliendo dicho mandato, el Real Decreto Legislativo 1/1994, de 20 de junio, por el que se aprueba el Texto Refundido de la Ley General de la Seguridad Social, no incorporó un capítulo dedicado a la asistencia sanitaria, consumándose así la existencia de un ordenamiento jurídico de la asistencia sanitaria pública, separado y distinto del de la Seguridad Social ${ }^{4}$.

Sin embargo, en la práctica la asistencia sanitaria siguió dependiendo de la Seguridad Social, y cuando en 1995 se aprueba el RD 63/1995, de 20 de enero, de ordenación de las prestaciones sanitarias del SNS, el mismo se dicta al amparo tanto del art. 149.1.16 $6^{\underline{a}}$ CE (Sanidad) como del 149.1.17 ${ }^{\mathrm{a}} \mathrm{CE}$ (Seguridad Social). Por lo tanto, pese a las determinaciones de la LGS, el SNS no queda claramente deslindado de la Seguridad Social. Pero, es más, con la Ley 24/1997, de 15 de julio, de consolidación y racionalización del Sistema de Seguridad Social, se llega a un nuevo punto de confusión, al integrarse nuevamente la asistencia sanitaria en los casos de maternidad, enfermedad común o profesional, y accidentes, sean o no de trabajo, en la acción protectora de la Seguridad Social. Confusión definitivamente consagrada cuando se modifican los Estatutos de las CCAA de "vía lenta" para que las correspondientes CCAA

\footnotetext{
${ }^{3}$ Esta afirmación queda corroborada por lo dispuesto en el artículo 44 y 45 de la LGS. En el primero se prevé que "todas las estructuras y servicios públicos al servicio de la salud integrarán el SNS". Y en el segundo se dispone que "el SNS integra todas las funciones y prestaciones sanitarias que, de acuerdo con lo previsto en la presente Ley, son responsabilidad de los poderes públicos para el debido cumplimiento del derecho a la protección de la salud".

Del mismo modo, la disposición adicional sexta de la LGS establece que "los centros sanitarios de la Seguridad Social quedarán integrados en el Servicio de Salud en los casos en que la Comunidad Autónoma haya asumido competencias en materia de asistencia sanitaria de la Seguridad Social, de acuerdo con su Estatuto de Autonomía. En los restantes casos, la red sanitaria de la Seguridad Social se coordinará con el Servicio de Salud de la Comunidad Autónoma”. Y la transitoria tercera que "el Instituto Nacional de la Salud continuará subsistiendo y ejerciendo las funciones que tiene atribuidas en tanto no se haya culminado el proceso de transferencias a las Comunidades Autónomas con competencia en la materia".

${ }^{4}$ Esta conclusión es alcanzada con bastante unanimidad por la doctrina a la luz del marco legislativo descrito. Así, Alonso Olea reconoce "la tendencia evidente a la independencia de la asistencia sanitaria respecto del complejo de la Seguridad Social", en Las Prestaciones del Sistema Nacional de Salud, Civitas, 1999, págs. 19-23.
} 
asuman las competencias de asistencia sanitaria de la Seguridad Social; o cuando, por la Ley 29/2001, de 27 de diciembre, se modifica el artículo 86.2 del Texto Refundido de la Ley General de la Seguridad Social de 1994, para dejar bien sentado que la asistencia sanitaria tiene naturaleza no contributiva, pero que queda incluida en la acción protectora de la Seguridad Social.

Así pues, el ordenamiento jurídico no definía claramente el sistema en el que se debía integrar la asistencia sanitaria, pues, pese a que la CE establece un modelo abierto y, sobre todo, al pronunciamiento expreso y clarificador de la LGS de 1986, que integra la asistencia sanitaria en el SNS, lo cierto es que cada una de las diferentes normas que se han ido dictando han integrado la asistencia sanitaria en la Seguridad Social. Con lo que, desde una perspectiva normativa, en torno a esta cuestión asistimos a un auténtico totum revolutum.

No puede extrañarnos que ante esta circunstancia la doctrina haya optado por interpretaciones muy posibilistas. Es el caso de Alarcón Caracuel, quien habla de relativa independización de la asistencia sanitaria respecto de la Seguridad Social: la asistencia sanitaria se presta por el Sistema Nacional de Salud, la cual es una parte distinta e independiente del Sistema de Seguridad Social, pero resulta que a las prestaciones del Sistema Nacional de Salud se puede acceder en virtud de diversos títulos: uno de ellos pertenecer al campo subjetivo de protección del Sistema de Seguridad Social, ya que entre sus prestaciones figura la asistencia sanitaria ${ }^{5}$. Por su parte, Pemán Gavín, también a partir de la Ley 24/1997 citada, considera que el modelo de servicio de salud universalizado puede calificarse formalmente como Sistema Nacional de Salud o como prestación incluida en un "tramo" de la Seguridad Social que se configura con carácter no contributivo y universalizado ${ }^{6}$. En fin, Beltrán Aguirre $^{7}$ argumenta que no se termina de integrar la asistencia sanitaria en el Sistema Nacional de Salud, y por ende, en el título competencial y en la materia de Sanidad, porque al Estado no le interesa. Afirma que "la reforma de los Estatutos de Autonomía de las CCAA del artículo 143 CE da a entender que

\footnotetext{
5 "Veinte años de ordenamiento laboral y de la Seguridad Social conforme a la Constitución", en el libro colectivo, 20 Años de Ordenamiento Constitucional, Aranzadi, 1999, p. 67

6 "Del Seguro Obligatorio de Enfermedad al Sistema Nacional de Salud. El cambio en la concepción de la asistencia sanitaria pública durante el último cuarto del siglo XX", Revista Aragonesa de Administración Pública, núm.13, 1998, p. 95.

7 "Prestaciones sanitarias y autonomías territoriales: cuestiones en torno a la igualdad". Revista de Derecho y Salud, Vol. 10, Núm. 1, Enero-Junio 2002.
} 
quiere que cuelgue del título competencial Seguridad Social, título que permite mayor control e intervención de la política asistencial de las CCAA toda vez que comprende la competencia exclusiva sobre el régimen económico de la Seguridad Social".

Esta última visión realista describe con precisión la dialéctica subyacente a nuestro ordenamiento. Hay que decir por ello en este sentido que en principio, nada impide que toda la organización prestacional sanitaria se desgaje definitivamente del Sistema de Seguridad Social y se integre en el Sistema Nacional de Salud, que prestará la asistencia sanitaria a todos los ciudadanos por el simple hecho de serlo, y con más razón cuando, además, se sea beneficiario de la Seguridad Social. En suma, nada impide que la asistencia sanitaria pueda colgarse en exclusiva de la materia y título competencial Sanidad.

Esta era la línea que -superado el cambio de rumbo sobre lo previsto por la LGS de 1986, al que nos hemos referido- parecía estaba a punto de consolidarse con ocasión de la aprobación de la reciente LCCSNS. En efecto, el citado cambio de rumbo respondía a un dato sustantivo: la financiación por la Seguridad Social. Por ello mismo, la progresiva financiación de la asistencia sanitaria pública vía presupuestos generales en lugar de vía cotizaciones sociales, culminada en el año 2000, la asunción definitiva por todas las CCAA de las competencias en asistencia sanitaria, el traspaso a su favor de los servicios, centros y funciones en el año 2001, así como el nuevo modelo de financiación sanitaria de las CCAA aprobado por la Ley 21/2001, de 27 de diciembre, son hechos que parecían propiciar la desvinculación definitiva de la asistencia sanitaria de la Seguridad Social. Y en efecto, esta intención quedó plasmada en el proyecto de LCCSNS, que invocaba como único título competencial el artículo 149.1.16 $6^{\mathrm{a}}$ (Sanidad) de la CE y se refería reiteradamente a la integración, unidad y cohesión del Sistema Nacional de Salud.

Sin embargo, el texto de la LCCSNS definitivamente promulgada invoca como títulos competenciales tanto el art. 149.1.16 $6^{\mathrm{a}}$ como el art. 149.1.17 $\mathrm{a}$ de la CE. Con ello puede decirse que la LCCSNS no ha modificado en este punto la situación preexistente. Es más, que el modelo de la LGS de 1986 y la construcción propia del Sistema Nacional de Salud que al menos formalmente ésta diseñó podrían así quedar en entredicho. Es muy posible que la LCCSNS al invocar como título competencial el artículo 149.1.17 a CE, y no sólo el 149.1.16 $6^{\mathrm{a}} \mathrm{CE}$, haya buscado tener un "plus" competencial para garantizar la cohesión sanitaria dentro del marco de descentralización de los servicios sanitarios en el que ya nos encontramos. Pero de lo que no cabe ninguna duda es que todavía hoy, tras casi dos décadas desde la LGS de 1986, 
persiste el asistematismo y la confusión entre el Sistema Nacional de Salud y la Seguridad Social.

Sigue siendo, pues, un "desideratum" el adecuado reparto sistemático al respecto: para la Seguridad Social la organización y gestión de las prestaciones económicas, debiendo las asistenciales cobrar autonomía respecto de la Seguridad Social y asentarse en organizaciones públicas diferentes especializadas en esta función asistencial, concretamente, en nuestro caso, los Servicios Autonómicos de Salud integrados en el Sistema Nacional de Salud, cuya organización y desenvolvimiento debiera tener lugar en el marco del título competencial Sanidad, no en el de Seguridad Social. "Desideratum", en efecto, si tenemos en cuenta que hoy en día ya no tiene sentido seguir encuadrando la asistencia sanitaria en el Sistema de la Seguridad Social, máxime cuando los principios rectores del sistema sanitario público son la universalidad y la equidad en el acceso a los servicios sanitarios, principios que se garantizan desde la integración y unidad del sistema, en definitiva desde el Sistema Nacional de Salud, al amparo del art. 149. $116^{\mathrm{a}}$ de la CE.

\section{LAS PRESTACIONES DEL SISTEMA NACIONAL DE SALUD}

La LCCSNS regula la ordenación de las prestaciones sanitarias. A tal fin define el Catálogo de Prestaciones como el conjunto de servicios preventivos, diagnósticos, terapéuticos, rehabilitadores y de promoción de la salud dirigidos a los ciudadanos que garanticen las condiciones básicas y comunes para una atención integral, continuada y en el nivel adecuado de atención. El Catálogo comprende las prestaciones de salud pública, atención primaria, atención especializada, atención sociosanitaria, atención de urgencias, prestación farmacéutica, la ortoprotésica, de productos dietéticos y de transporte sanitario (art. 7 de la Ley).

Estas prestaciones se hacen efectivas a través de un conjunto de técnicas, tecnologías y procedimientos que integran la Cartera de Servicios del Sistema Nacional de Salud, la cual será acordada en el seno del Consejo Interterritorial del Sistema Nacional de Salud, y aprobada por Real Decreto. Su actualización se aprobará por Orden del Ministro de Sanidad y Consumo, previo acuerdo del Consejo Interterritorial del Sistema Nacional de Salud. Cada Comunidad Autónoma, por su parte, podrá aprobar su propia cartera de servicios, que incluirá como mínimo la cartera de servicios del Sistema Nacional de Salud (art. 20). En este sentido, hay que subrayar la inclusión de la salud pública y la atención sociosanitaria como prestaciones sanitarias. 
1. En esta nueva regulación se observa una mayor ordenación y definición de las actividades de salud pública, a diferencia de lo que ocurría en la Ley General de Sanidad, en la que estaban totalmente dispersas a lo largo del articulado. La salud pública se configura ahora como prestación sanitaria, encaminada a preservar, proteger y promover la salud de la población, a través de actuaciones dirigidas, entre otras finalidades, a la información y vigilancia epidemiológica, a la protección y promoción de la salud, la prevención de las enfermedades y de las deficiencias, la vigilancia y control de los posibles riesgos para la salud derivados de la importación, exportación o tránsito de mercancías y del tráfico internacional de viajeros, la protección y promoción de la salud ambiental y laboral, y la promoción de la seguridad alimentaria (art. 11). La LCCSNS asume así una concepción integral de la sanidad, ya defendida por la Ley General de Sanidad, pero que ahora se concibe desde una clara voluntad de integración.

2. La asunción por todas las CCAA en 1987 de las competencias en la materia específica de salud pública (sanidad e higiene), había dado lugar a la existencia de subsistemas totalmente independientes de salud pública, con una evidente falta de coordinación entre las Administraciones públicas sanitarias que se ha hecho patente en especial con ocasión de la necesaria adopción de decisiones de interés general en momentos difíciles y proclives a la alarma social (por ejemplo, los casos de las "vacas locas" o los supuestos de "meningitis C"), lo que ha dado lugar a la existencia de subsistemas totalmente independientes de salud pública. Por ello, la LCCSNS dedica todo un capítulo de la misma a regular la actuación coordinada en salud pública y seguridad alimentaria, así como al establecimiento de un Plan de cooperación y armonización de actuaciones en el ámbito de la salud pública (art. 65 y 66).

Esa actuación coordinada de las Administraciones Públicas Sanitarias en salud pública se prevé para dos supuestos: a) ante situaciones de especial riesgo o alarma para la salud pública; y b) para dar cumplimiento a acuerdos internacionales, así como a programas derivados de las exigencias de la normativa europea, cuando su cumplimiento y desarrollo deban ser homogéneos en todo el Estado.

Por su parte, el Plan de cooperación y armonización de actuaciones en el ámbito de la salud pública, aunque el legislador no lo define expresamente, viene a ser un instrumento de planificación sanitaria para garantizar la cooperación entre las Administraciones autonómicas y locales, y cuya finalidad es promover actividades que complementen las realizadas por dichas Administraciones públicas. Así, dicho Plan tiene que: a) establecer las funciones bási- 
cas en materia de salud pública a desarrollar en todo el Estado, fundamentadas en el análisis de la situación de salud y en las estrategias y compromisos adquiridos en el ámbito internacional, de acuerdo con la evidencia científica disponible; b) definir la cartera de servicios y las garantías correspondientes a dichos servicios; c) establecer los medios y sistemas de relación entre las Administraciones públicas para facilitar la información recíproca y el seguimiento del plan; d) facilitar la promulgación de la legislación sanitaria y la aplicación de las directivas y reglamentos de la Unión Europea que afectan a la salud pública; y e) promover el desarrollo de hábitos de colaboración y participación en los que se sustente la práctica profesional.

3. Como decíamos, debe resaltarse la inclusión por la Ley dentro de las prestaciones sanitarias de la atención sociosanitaria. La misma queda configurada como una prestación del Sistema Nacional de Salud que comprende el conjunto de cuidados destinados a aquellos enfermos, generalmente crónicos, que por sus especiales características pueden beneficiarse de la actuación simultánea de los servicios sanitarios y sociales para aumentar su autonomía, paliar sus limitaciones o sufrimientos y facilitar su reinserción social. Comprende los cuidados de larga duración, la atención sanitaria a la convalecencia y la rehabilitación en pacientes con déficit funcional recuperable (art. 14).

Entendiendo la atención sociosanitaria como aquella atención especial e integral dirigida a personas con necesidad de atención "social" y de "salud" de manera permanente aunque con intensidades variables, el problema reside en la coordinación entre los servicios sociales y de salud, máxime cuando se integra la atención sociosanitaria en el Sistema Nacional de Salud sin tener hoy por hoy una Ley de servicios sociales sobre la Dependencia, de carácter estatal, que delimite el ámbito de actuación de las distintas Administraciones públicas (Estado, CCAA y Administración Local), coordine los diferentes sectores, y-lo que es más importante- regule y solucione los problemas que esa coordinación plantea.

En este sentido destaca como principal problema, a nuestro entender, la diferente configuración de la sanidad y los servicios sociales, ya que la sanidad tiene el carácter de prestación universal y gratuita, caracteres éstos de los que adolecen los servicios sociales, pues hasta este momento, los usuarios de los mismos han tenido que participar en la financiación. Por otra parte, nos preguntamos cómo ha de entenderse la coordinación de la que habla la Ley y sobre todo cómo será la coordinación financiera, es decir, si toda prestación de asistencia sociosanitaria se financiará vía presupuestos generales al igual que la asistencia sanitaria, y si así fuera, qué parte lo sería con cargo a los presupuestos destinados a sanidad 
y cuál a los destinados a servicios sociales. Es más, si no debería optarse por un sistema mixto con intervención de la iniciativa privada ó asumir un seguro estatal de cobertura de los riesgos por dependencia.

Entendemos que la financiación pública debiera ser mayor cuanto mayor sea el nivel de dependencia que tuviese el individuo o más débil fuese su situación económica. Habría así una cobertura universal para las situaciones de gran dependencia, que se conseguiría mediante impuestos generales. Dentro de este nivel de gran dependencia se incluirían aquellos individuos que tienen necesidad de apoyo continuado durante mucho tiempo para llevar a cabo las actividades de la vida diaria (hay que tener en cuenta que por lo general estos casos precisan de internamiento, y el nivel de dependencia está asociado a un conjunto de problemas de salud de carácter crónico).

4. Una última consideración sobre la nueva regulación de las prestaciones sanitarias del Sistema Nacional de Salud a destacar es que la Cartera de Servicios se aprobará con carácter reglamentario, es decir, con rango de Real Decreto, -y su actualización mediante Orden-, lo que plantea si no habría sido más correcto jurídicamente que fuera aprobada con rango legal. Se ha optado por el reglamento por entender que la Cartera de Servicios es una norma que desarrolla la LCCSNS, y que encuentra en la misma la habilitación legal necesaria. Sin embargo, la regulación de la Cartera de Servicios es una normación básica, ya que establece el mínimo común para todo el Estado a partir del cual las CCAA podrán regular su propia Cartera de Servicios, lo que suscita la cuestión de su eventual necesaria regulación por norma legal ${ }^{8}$, al menos en lo que respecta a las condiciones básicas y mínimas que garanticen la igualdad en el ejercicio de los correspondientes derechos prestacionales.

\footnotetext{
${ }^{8}$ Esta afirmación viene respaldada por la reiterada doctrina del Tribunal Constitucional sobre la noción de bases y sus elementos formales y materiales. Por ejemplo, y conforme a la sentencia del TC 69/1988, de 19 de abril: “... Es materialmente básica por garantizar en todo el Estado un común denominador normativo dirigido a asegurar, de manera unitaria y en condiciones de igualdad, los intereses generales a partir del cual puede cada Comunidad Autónoma, en defensa de sus propios intereses, introducir las peculiaridades que estime convenientes y oportunas dentro del marco competencial que en la materia le asigne su Estatuto". "A la segunda finalidad atiende el principio de Ley formal que la misma doctrina acoge en razón a que sólo a través de este instrumento normativo se alcanzará, con las garantías inherentes al procedimiento legislativo, una determinación cierta y estable de los ámbitos respectivos de ordenación de las materias en las que concurren y se articulan las competencias básicas estatales y las legislativas y reglamentarias autonómicas"... "Como excepción a dicho principio de Ley formal la referida doctrina admite que el Gobierno de la Nación pueda hacer uso de su potestad reglamentaria para regular por Decreto
} 


\section{LA COORDINACIÓN Y LA COOPERACIÓN EN EL SNS: EL CONSEJO INTERTERRITORIAL Y LA ALTA INSPECCIÓN}

En todo caso, el elemento central de la nueva Ley de Cohesión y Calidad es la regulación de la coordinación y la cooperación como ejes fundamentales del Sistema Nacional de Salud para garantizar así la existencia de un sistema sanitario público cohesionado y de calidad.

1. La coordinación general sanitaria queda consagrada en el art. 149.1.16 CE como una competencia exclusiva del Estado. Ya en la Ley General de Sanidad de 1986 se recogía la coordinación y la cooperación como funciones imprescindibles en un Sistema Nacional de Salud descentralizado y armónico. Sin embargo, y a pesar de que dicha Ley preveía mecanismos de cooperación y coordinación de carácter general -como el Consejo Interterritorial del Sistema Nacional de Salud, los convenios interadministrativos, los Planes de Salud y los Criterios generales de coordinación- los objetivos de integración que tales mecanismos persiguen no se han conseguido hasta la fecha de una manera adecuada.

Todo ello por razones diversas. En primer lugar, la composición y funcionamiento del Consejo Interterritorial del Sistema Nacional de Salud impedía que se convirtiera en el órgano fundamental del Sistema y encargado de su vertebración funcional. Por otra parte, los Criterios generales de coordinación, recogidos en el art. 70 LGS, no han sido aprobados por el Estado en el sentido establecido por la jurisprudencia del TC que equipara la coordinación con las bases en cuanto a su formalización, por lo que la existencia y exigibilidad de esos criterios precisaría su aprobación por órgano competente y su ulterior publicación en diario oficial, algo que sigue sin producirse. Por lo demás, tampoco se ha producido la aprobación del Plan Integral de Salud, previsto como el conjunto de Planes tanto estatales como autonómicos y que, recogiendo los Criterios generales de coordinación, sería el máximo instrumento de planificación coordinada entre todas las Administraciones públicas sanitarias.

\footnotetext{
alguno de los aspectos básicos de una materia, cuando resulten, por la naturaleza de ésta, complemento necesario para garantizar el fin a que responde la competencia estatal sobre las bases. Esta excepción... debe entenderse limitada al sentido que corresponde a su naturaleza de dispensa excepcional de suficiencia de rango normativo, pero no alcanza a la exigencia de que su carácter básico se declare expresamente en la norma o se infiera de su estructura en la misma medida en que ello es aplicable a la Ley formal, pues lo contrario sería permitir que por vía reglamentaría se introduzcan elementos de confusión e incertidumbre, siendo que ello se deja negado en la Ley formal".
} 
Por ello, buena parte de la cooperación y coordinación a lo largo de los últimos años, más de 16 tras la LGS, se ha llevado a cabo al margen de dichos mecanismos legales y de una manera en cierto sentido informal. De ahí que, en consecuencia, y de manera acuciante tras la culminación de la descentralización sanitaria en diciembre de 2001, se hiciera imprescindible la búsqueda de un nuevo modelo que garantizara un funcionamiento cohesionado del Estado y las CCAA en materia sanitaria, la modernización del Sistema Nacional de Salud que el entorno exige, y el disfrute por los ciudadanos de un servicio sanitario público de calidad y en condiciones de igualdad efectiva en el acceso al mismo independientemente del lugar de su residencia.

A esa necesidad intenta responder la recién promulgada Ley de Cohesión y Calidad del Sistema Nacional de Salud (no hay que olvidar que en sus borradores llevaba el título de Ley de Coordinación Sanitaria), cierto que con mucha distancia entre la gran virtualidad que a dicha coordinación le otorga su Exposición de Motivos al recoger la finalidad y los objetivos de la Ley, y las concretas previsiones de su articulado sobre dicha coordinación sanitaria, donde el entusiasmo por parte del legislador resulta mucho menos palpable, para lo cual configura el Consejo Interterritorial del SNS y la Alta Inspección como los ejes sobre los que girará tanto la coordinación como la cooperación de las Administraciones sanitarias públicas.

2. La LCCSNS define el Consejo Interterritorial del SNS como órgano permanente de coordinación, cooperación, comunicación e información. La Ley parece con ello haber optado por una configuración funcional de este órgano mucho más ambiciosa que la hasta ahora vigente (el Consejo Interterritorial del SNS se definía en la Ley General de Sanidad como órgano tan sólo coordinador entre las CCAA y la Administración General del Estado, y en el proyecto de la propia LCCSNS como órgano de cooperación). Resulta por ello oportuno que nos detengamos en el significado y alcance de los principios de coordinación y cooperación ${ }^{9}$.

La coordinación, a la que expresamente se refiere la CE, ha sido definida por la doctrina como un principio en virtud del cual se conjugan para una misma finalidad diversas acciones o diferentes entidades político-administrativas y cuyo fundamento radica en la supremacía de la propia instancia coordinadora. Como notas definitorias de la coordinación se destacan: implicar el

\footnotetext{
${ }^{9}$ Vid. José M. Souvirón Morenilla, Los elementos esenciales del sistema constitucional de organización territorial del Estado y su concreción, Comares, 2000, págs. 243-249.
} 
ejercicio separado, aunque coordinado, de sus propias competencias por cada una de las entidades de que se trate; tener un carácter vinculante derivado de la ley o norma superior, requisito necesario éste al constituir un límite al ejercicio de las competencias propias; afectar a la forma -no al fondo o contenido-de ejercitar las competencias, y habilitante por tanto para imponer un método de actuación, un modo de ejercer poderes propios y ajenos mediante la participación de las diversas instancias en la determinación de los objetivos y de los medios, pero sin que éstos como tales puedan imponerse.

Por su parte, la cooperación en sentido estricto implica un ejercicio conjunto de las competencias de diversas entidades jurídicos-públicas, cualquiera que sea el grado de esa conjunción y, como regla, con carácter voluntario y en posición de igualdad entre las partes, no afectando a la titularidad de las competencias en cuestión, sino al modo conjunto de su ejercicio. A diferencia del principio de coordinación, la cooperación no se encuentra recogida en el Texto constitucional, pero dado su alcance para la integración del Estado de las Autonomías, tanto la doctrina, como la jurisprudencia y el legislador ordinario han intentado fundamentarla desde diversas perspectivas. Así, para MUÑOZ MACHADO10 "la cooperación resulta del hecho de que la mayor parte de las competencias se distribuyen en régimen de compartición, y por tanto no pueden ejercerse eficazmente si no hay entendimiento entre los entes responsables (...) la CE no la impone (la cooperación), pero su sistema la implica”. Por su parte, el TC ha visto en el principio de cooperación un dato implícito en el Estado Autonómico, derivado del deber general de colaboración, al que, más allá del efecto limitante en lo formal de la coordinación y de las obligaciones de recíproca información y auxilio derivadas de la colaboración en sentido estricto, vendría a culminar, pero sobre la base de la voluntad de las partes implicadas ${ }^{11}$. En cuanto al legislador ordinario, éste contempló la

${ }^{10}$ Derecho público de las Comunidades Autónomas, vol. I, pág. 221.

11 "Principio de cooperación que ha de regir las relaciones entre entres públicos territoriales en nuestro ordenamiento"(STC 157/85, FJ 2), en tanto que desideratum del Estado autonómico ("la deseable cooperación de todas las autoridades interesadas en la rápida solución del problema"-STC 33/82, FJ 4- ; "la conveniencia de una cooperación entre el Estado y las Comunidades Autónomas en beneficio recíproco" -STC 71/83, FJ 3 y 4-). "No significa esto en modo alguno que cuando el Estado carece de competencia para la coordinación general, la consecución de ese objetivo se hace imposible, pues el Estado y las Comunidades Autónomas están sometidas recíprocamente a un deber general de colaboración (...) Pero como este deber no implica extensión alguna de las competencias estatales, el Estado no puede tratar de imponerlo mediante la adopción de medidas coercitivas, sino buscando para la que haya de adoptar previa conformidad de las Comunidades Autónomas competentes, que, por esta vía, participan en la formación de la voluntad general del Estado" (STC 80/85, FJ 3). 
cooperación en lo que respecta a las relaciones de los entes locales con el Estado o las Comunidades Autónomas en la LBRL; y en lo que se refiere a las Comunidades Autónomas, diversas leyes sectoriales, como por ejemplo, los mecanismos de cooperación en materia sanitaria previstos por la propia LGS de 1986. En fin, y referida a la cooperación vertical entre el Estado y las Comunidades Autónomas la Ley 4/1999, por la que se reformó la LRJ-PAC, incluye ya un concepto estricto de cooperación cuando contempla las relaciones que tengan como finalidad la toma de decisiones conjuntas que permitan, en aquellos asuntos que afecten a competencias compartidas o exijan articular una actividad común entre ambas Administraciones, una actividad más eficaz de los mismos (art. 4.5).

Pues bien, a partir de lo expuesto, resulta claro que el cambio textual operado por la LCCSNS en la definición del Consejo Interterritorial del SNS sobre la precedente definición de la LGS responde a su voluntad de ajustar el protagonismo del mismo que se pretende, a una definición estricta de los principios de integración funcional concernidos y que ahora específicamente se enumeran como la base de acción de dicho Consejo. Por tanto, con una precisión técnica que supera los equívocos precedentes tanto de la LGS, que reconducía la acción del Consejo Interterritorial del SNS tan sólo a la coordinación -mal entendida-, como del propio TC, incurso en el mismo equívoco ${ }^{12}$.

El protagonismo que se pretende para el Consejo Interterritorial del SNS se reconoce asimismo en la composición de dicho Consejo establecida por la LCCSNS comparada con lo dispuesto en la Ley General de Sanidad. Ahora estará integrado por el Ministro de Sanidad y Consumo, que lo presidirá, los Consejeros competentes en materia de sanidad de las CCAA y un secretario, que será un representante del Ministerio de Sanidad y Consumo, sin perjuicio, de que cuando sea necesario se puedan incorporar representantes del Estado o de las CCAA afectadas (art. 70).

Por otra parte, y bajo el mismo designio, la nueva Ley precisa el elenco de funciones que debe ejercer el Consejo y que, recogidas en su artículo 71, son clasificadas por el legislador en cuatro grupos: a) funciones esenciales en la

\footnotetext{
12 El TC, en sus sentencias 32/1983 y 42/1983, establecía que "la coordinación sanitaria general debe ser la fijación de medios y sistemas de relación que hagan posible la información recíproca, la homogeneidad técnica y la acción conjunta de las Administraciones públicas sanitarias en el ejercicio de sus respectivas competencias, de tal modo que se logre la integración de actos parciales en la globalidad del Sistema Nacional de Salud".
} 
configuración del Sistema Nacional de Salud; b) funciones de asesoramiento, planificación y evaluación en el Sistema Nacional de Salud; c) funciones de coordinación del Sistema Nacional de Salud; y d) funciones de cooperación entre el Estado y las CCAA. En todo caso, la densa sistemática y la casuística de la Ley en la ordenación de las funciones del Consejo decae ante el problema de la ubicación de cada una de las competencias del Consejo en uno u otro de tales grupos específicos de funciones (y de los principios funcionales a que respectivamente responden). Lo que, en concreto, suscita el problema de cómo y con qué alcance tiene que funcionar el Consejo en el ejercicio de cada una de sus competencias. Por ejemplo, la aprobación de los planes integrales se encuentra dentro del primer grupo, es decir dentro de las funciones esenciales en la configuración del Sistema Nacional de Salud, y siendo así, ¿el Consejo funcionará a ese respecto como órgano de coordinación, cooperación, comunicación o información?

Otro ejemplo, el Consejo, y para todas y cada una de sus funciones, dice la Ley, que "conocerá, debatirá y, en su caso, emitirá recomendaciones" (art. 71), previendo el artículo 73 que los acuerdos del Consejo se plasmarán a través de recomendaciones que se aprobarán por consenso. No hay duda que estas determinaciones restan fuerza al papel del Consejo dentro del Sistema Nacional de Salud porque quedan así excluidas la obligatoriedad y fuerza vinculante que deberían tener sus decisiones para que nuestro sistema sanitario público fuera un sistema armónico, cohesionado y de calidad. Del mismo modo, ese carácter obligatorio y vinculante queda de suyo relativizado en las relaciones entre la Administración sanitaria central y las autonómicas, al articularlas la Ley bajo el principio de cooperación, y por ende el de la voluntariedad de las Administración públicas implicadas.

En cuanto al funcionamiento del Consejo, habrá que estar en concreto a lo que disponga su reglamento de organización y funcionamiento, aún no aprobado, el cual -entendemos- debería apostar definitivamente por una potenciación de su intervención dentro del sistema, abandonando así el papel desempeñado hasta el momento, más bien de órgano consultivo y de foro de intercambio y debate entre los responsables de los distintos servicios de salud, autonómicos y estatal para, más allá de la vía amigable y consensuada en la toma de decisiones, caminar por el reconocimiento a las mismas de un efecto vinculante y la consecuente formulación de algún mecanismo para su publicación en los Diarios Oficiales.

Son, pues, diversos los problemas que plantea la configuración del Consejo Interterritorial del SNS, problemas tampoco solubles desde su eventual ti- 
pificación orgánica ${ }^{13}$. Lo que no excluye la unanimidad en considerar que el Consejo Interterritorial del SNS debería convertirse en el foro imprescindible para desarrollar el diálogo entre los diversos agentes implicados y adoptar las decisiones fundamentales para el sistema sanitario.

3. En lo que se refiere a la Alta Inspección, no hay en cuanto a las funciones y al procedimiento de actuación grandes diferencias entre lo establecido en la nueva LCCSNS y lo previsto en la Ley General de Sanidad. Es más, aunque aquélla deroga el artículo 43 de la LGS, la LCCSNS viene, sin embargo, a copiar literalmente dicho precepto en su artículo 76. Su funcionamiento parece, pues, va a cambiar poco con respecto a la situación anterior, cuando, aun partiendo del obligado perfil limitado que a dicha Alta Inspección ha reconocido el $\mathrm{TC}^{14}$, entendemos debería potenciarse su funcionamiento y re-

\footnotetext{
13 Ante la escasa regulación de este órgano, el profesor Villar Rojas ("Análisis de la cooperación en un sector determinado: la sanidad pública”. Revista de Derecho y Salud, Vol. 10, Núm. 1, Enero-Junio, 2002), ya antes de la LCCSNS, reflexiona sobre su naturaleza jurídica, en esencia sobre si estábamos ante un ejemplo particular de Conferencia Sectorial o ante un órgano colegiado estatal con representantes autonómicos, para concluir que se trataba de una organización singular, sobre la base de los siguientes datos: su creación se produce "ope legis", no por acuerdo interadministrativo, como ocurre con las Conferencias Sectoriales (art. 5.2 de la Ley 30/1992, de 26 de noviembre, de Régimen Jurídico de las Administraciones Públicas y del Procedimiento Administrativo Común); no es un órgano de la Administración Central, sino que lo es del Sistema Nacional de Salud, aunque la Secretaria Permanente del Consejo, que le sirve de soporte administrativo y de vehículo de comunicación entre sus miembros, sí que es un órgano integrado en el Ministerio de Sanidad; los acuerdos que adopta no vinculan las competencias autonómicas ni estatales, salvo que las asuman como propias, igual que ocurre con las Conferencias Sectoriales, pero, el modo de llegar a esos acuerdos es peculiar: se aprueban por asentimiento de los miembros, siempre que ninguno exprese su parecer contrario, y sin que exista un mecanismo ni de formalización, ni de publicación de esos acuerdos; a pesar de presentarse en la LGS como un órgano que puede ejercer competencias de coordinación y planificación, a lo largo de los años ha desarrollado una función análoga a las Conferencias Sectoriales, es decir, que ha ejercido un papel de "amigable componedor". Tras todo esto concluye Villar Rojas que "el CISNS cumple, esencialmente, el papel de órgano consultivo de las autoridades sanitarias estatales, con la participación de las CCAA, siendo la legislación estatal la que se debate. Más que una Conferencia Sectorial, recuerda a un órgano colegiado estatal con participación autonómica (art. 5.1, segundo párrafo de la Ley de Procedimiento Administrativo Común). Estas dudas, sin embargo, no deben llevar a equívoco. Ni la dificultad para encasillar a este órgano, ni su insuficiente régimen jurídico, pueden ocultar una realidad indiscutible: desde su constitución, el CISNS ha cumplido su función como lugar de intercambio y debate entre los distintos responsables de los distintos servicios de salud autonómicos y estatal".
}

${ }^{14}$ El TC ha afirmado que la Alta Inspección del Estado no constituye un control jerárquico o tutelar, sino que, dirigida a garantizar la correcta interpretación de la norma, conlleva tan sólo una potestad de vigilancia, de verificación o fiscalización que pueda llevar en su caso a instar la 
definirse su actuación dentro del Sistema sanitario, como una inspección colaborativa, cuyos objetivos sean la garantía para el ciudadano de la equidad y de la calidad y de su participación democrática en los servicios sanitarios. Y a cuyo fin podría, entre otras, desarrollar las funciones de: verificación de las garantías de las prestaciones del Sistema Nacional de Salud en el ámbito de las CCAA; seguimiento y análisis de los actos y disposiciones de las CCAA; seguimiento de la ejecución de los convenios adoptados en el seno del Consejo Interterritorial del SNS, y de los acuerdos suscritos por el Ministerio de Sanidad y Consumo con otras Administraciones públicas y entidades públicas o privadas; supervisar el desarrollo y adecuación en las CCAA de los planes integrales; o vigilar la correcta aplicación de las normas estatales en su ejecución por las CCAA.

Supone en cambio una novedad de la LCCSNS la regulación de la coordinación y la cooperación de la inspección en el Sistema Nacional de Salud. La Alta Inspección del Estado establecerá mecanismos de coordinación y cooperación con los servicios de inspección de las CCAA, en especial en lo referente a las actuaciones dirigidas a impedir o perseguir todas las formas de fraude, abuso, corrupción o desviación de las prestaciones o servicios sanitarios con cargo al sector público, cuando razones de interés general así lo aconsejen.

4. Finalmente, conviene destacar como en la nueva Ley no se recoge ya dentro de la planificación sanitaria la elaboración de un Plan Integrado de Salud, que en la LGS se definía como el documento único en el que se recogerían los planes estatales, los planes de las CCAA y los planes conjuntos, teniendo en cuenta los criterios de coordinación general sanitaria. Ahora se acude a la fórmula de los Planes Integrales, opción plausible a la vista de la imposibilidad de elaborar un Plan Integral de Salud cuando hay CCAA que van ya por su segundo Plan de Salud, mientras otras ni siquiera han elaborado el primero.

Los Planes Integrales de Salud se regulan en el artículo 64 de la LCCSNS, conforme al cual tales Planes se elaborarán sobre las patologías más prevalentes, relevantes o que supongan una especial carga sociofamiliar, garantizando una atención sanitaria integral, que comprenda su prevención, diagnóstico, tratamiento y rehabilitación. Para ello, establecerán criterios sobre la forma de

activación de los controles sobre las Comunidades Autónomas constitucionalmente establecidos, básicamente de carácter jurisdiccional, pero no a la reacción a través de medidas concretas (STS $6 / 82$ y $32 / 83$ ). 
organizar los servicios para atender las patologías de manera integral y semejante en el conjunto del Sistema Nacional de Salud; determinarán los estándares mínimos y los modelos básicos de atención para la prevención, detección precoz, diagnóstico, tratamiento y rehabilitación de grupos de enfermedades; y especificarán actuaciones de efectividad reconocida, identificarán modelos de atención de estas intervenciones, desarrollarán herramientas de evaluación e indicadores de actividad, indicarán metas y objetivos para evaluar el progreso e identificarán insuficiencias en el conocimiento para orientar las prioridades de investigación.

\section{A MODO DE CONCLUSIÓN}

La aprobación de La LCCSNS era una necesidad evidente. Aún a sabiendas de que su puesta en práctica puede traer implicaciones no del todo esperadas (pues todavía falta el desarrollo reglamentario de sus preceptos), no hay duda de que, culminada la descentralización sanitaria y extinguido el INSALUD, era necesaria una regulación del papel a jugar por el Estado dentro de un modelo sanitario descentralizado, cohesionado y de calidad.

No es concebible, en efecto, la existencia de subsistemas sanitarios independientes unos de otros en los que el disfrute de los servicios sanitarios se delimite, no ya orgánica y funcionalmente, sino también cuantitativa y cualitativamente, tan sólo en función del criterio territorial, con el riesgo de que ello afecte a la adecuada prestación de los servicios y la satisfacción de los derechos ciudadanos en términos de garantía e igualdad.

El ciudadano tiene derecho a la asistencia sanitaria en todo el territorio nacional, pero la descentralización de los servicios puede dar lugar a situaciones en las que el disfrute de ese derecho quede territorialmente acotado e incluso condicionado. Por ello, son necesarias las fórmulas de cooperación y un ejercicio efectivo de las competencias del Estado y de su función de árbitro entre los servicios regionales de salud y entre éstos y los usuarios, sobre todo en algunos apartados fundamentales.

La descoordinación en materia normativa autonómica es hoy una de las grandes fallas de nuestro Sistema Sanitario, pues manifiesta la territorialidad del derecho que antes se apuntaba, resultando imprescindible poner en marcha instrumentos de coordinación normativa para un desarrollo jurídico armónico de los aspectos fundamentales del Sistema Nacional de Salud. 
De otra parte, y al margen de la asimetría en las regulaciones de los sistemas de salud autonómicos, quizás donde más patente ha sido esa falta de coordinación es en la adopción de decisiones importantes en momentos difíciles en actuaciones de salud pública (a algunos supuestos reales y suficientemente conocidos nos hemos referido más arriba), en los que cada una de las CCAA adoptó sus propias decisiones, sin establecerse un plan de cooperación para la actuación conjunta de las autoridades sanitarias.

Sin embargo, parece que el legislador continúa sin entender la necesidad de dar más fuerza a la coordinación y cooperación sanitaria, cuando es evidente que son estas figuras que caracterizan las relaciones entre las Administraciones públicas, las que si se regulan correctamente en el ámbito sanitario, conseguirían ser el centro del Sistema, y garantizar un Sistema Nacional de Salud cohesionado y armónico.

Dudamos, pues, que con la regulación que la LCCSNS hace de la coordinación y cooperación sanitaria pueda alcanzarse de un modo óptimo el objetivo de cohesión del Sistema Nacional de Salud. En este sentido resulta reveladora la confusa regulación del Consejo Interterritorial del Sistema Nacional de Salud, pues a pesar de establecerse una ordenada clasificación de sus funciones, no puede predecirse con certeza cuando y ante qué situaciones el mismo va a actuar o debe actuar como órgano de coordinación, de cooperación, de comunicación o de información. Por lo demás, sus decisiones continúan sin tener carácter obligatorio y fuerza vinculante para las Administraciones Públicas Sanitarias. Por otro lado, la débil regulación que se hace de la Alta Inspección impide que la coordinación y cooperación de las actuaciones sanitarias de las CCAA entre sí o con el Estado dispongan de ese necesario mecanismo final de garantía en términos satisfactorios. En suma, la LCCSNS se ha mostrado en exceso tímida para concretar lo que nos resulta un dato incuestionable: que la sanidad es un servicio público en el que la cooperación entre el Estado y las CCAA, y la coordinación en términos de superioridad del Estado para imponer condiciones, requisitos o criterios mínimos, son -o debieran ser-imprescindibles si se quiere asegurar la igualdad efectiva de todos en el derecho a la prestación de la salud. 